\title{
Critical tissue ischaemia in scleroderma: a note of caution
}

Vascular features dominate the clinical presentations of systemic sclerosis. Raynaud's phenomenon is the most typical presenting symptom. Renal involvement in early diffuse scleroderma and isolated pulmonary hypertension in late limited scleroderma are major causes of morbidity and mortality due exclusively to vascular change. A Raynaud-like transient vasoconstrictive response to cold has been suggested from clinical studies of the cardiac, pulmonary and renal circulations. ${ }^{1-3}$

The clinical phenomenology is traditionally attributed to the ubiquitous presence of a fibrotic arteriosclerotic lesion of varying severity. ${ }^{4}$ Physiological vasoconstriction superimposed on the fixed occlusive luminal hyperplasia is seen as an adequate explanation for transient ischaemia, that is, Raynaud's phenomenon, and should be viewed as amenable to therapy. Of more dire clinical consequence is irreversible occlusion with attendant critical tissue ischaemia and subsequent tissue injury.

There are few reliable data on factors involved in the initial pathogenesis and continuing evolution of the intimal hyperplasia and endothelial dysfunction which is typical of systemic sclerosis. There are virtually no data that explain the remarkable differences in clinical severity, pace of development, and patterns of visceral distribution of vascular injury between patients and classes of patients.

This lack of basic understanding should not encourage nihilism by the bedside clinician. While fibrotic vascular occlusion is currently an untreatable lesion, a variety of potentially controllable factors should be considered in either or both intermittent vasoconstriction and irreversible vascular occlusion.

For example, in vivo platelet activation is well documented in systemic sclerosis. Local adherence to damaged endothelium and circulating platelet aggregates as microemboli are two mechanisms for occlusion. Local release by activated platelets of thromboxane A2 and serotonin is a mechanism for vasoconstriction. ${ }^{5}$

Critical tissue ischaemia is a complex and multifactorial biological process. Many of the accepted participatory factors are well-documented in scleroderma including impaired vasomotion of afferent arterial circulation, enhanced neutrophil and platelet adherence to damaged endothelium, altered microcirculatory hydrostatics, and impaired venous outflow. Endothelial expression of ELAM-1 (endothelial leucocyte adherence molecule) is present in the microvasculature at very early stages of scleroderma $^{6}$ as are peri-intimal foci of adherent neutrophils. Irrespective of the mechanism of initial injury, the endothelial response includes a number of proconstrictive and/or prothrombotic elements including increased endothelin-1 release, ${ }^{7}$ diminished reserve of plasminogen activator ${ }^{8}$ and decreased production of endothelium dependent relaxing factor (nitric oxide).

The complexity of the process suggests that no single therapy is adequate. Iloprost, a carbaprostacyclin, is theoretically well suited for critical tissue ischaemia and has been demonstrated effective for severe Raynaud's phenomenon and digital ulcers complicating scleroderma, in both American and European studies..$^{9-11}$ Iloprost will not be marketed in the USA. Alternative prostanoids, including orally bioavailable formulations, remain investigational. A variety of promising new approaches to treatment are on the horizon including potent and specific thromboxane A2 receptor antagonists and endothelin-1 receptor antagonists.
One potentially treatable mechanism of vascular occlusion would be vasculitis. Other than in the setting of overlapping sicca syndrome, ${ }^{12}$ vasculitis of any sort or degree is considered uncommon in scleroderma. This issue contains two reports from Herrick et al suggesting the contrary. The first describes an unfortunate individual with long standing diffuse scleroderma who presented with clinical and histopathological evidence of diffuse vasculitis in overlap with active myositis. Clinical and laboratory studies were convincing enough to warrant aggressive glucocorticoid and cyclophosphamide therapy. ${ }^{13}$ Such an overt presentation of vasculitis in scleroderma is so rare, however, that it excludes an important cause and effect relationship.

The second report retrospectively surveys patients at their centre with digital ischaemia sufficiently severe to warrant surgical amputation. ${ }^{14}$ Vasculitis of diverse vascular location, histology and severity was present in five of nine subjects. Low levels of anticardiolipin antibody were present in four. Other than cigarette smoking in seven, these individuals are notable for their lack of commonality and their general lack of other clinical and laboratory features that suggested vasculitis.

The question inevitably arises as to the advisability of glucocorticoid and/or immunosuppressant therapy for major digital ischaemia presumably due to vasculitis. Glucocorticoid therapy at any level is associated with increased mortality in systemic sclerosis and with increased specific morbidity. The development of non-hypertensive renal failure in diffuse scleroderma receiving moderate glucocorticoid is but one example. ${ }^{15}$ In our own experience, individuals with isolated pulmonary hypertension are twice as likely as matched control subjects to have received glucocorticoids in the past. Data from the American studies of iloprost link glucocorticoid therapy to the development of digital tip ulcerations as well. ${ }^{9}$ Impairment of endothelial prostacyclin production by glucocorticoid is a likely mechanism although other prothrombotic effects need be considered. ${ }^{15} 16$

There are multiple plausible opportunities for intervention (table) in the patient with an ischaemically comprised digit. First and foremost is smoking cessation.

\begin{tabular}{ll} 
A hierarchy of treatment options for the ischaemically compromised digit \\
\hline Goal & Primary and secondary considerations \\
\hline IMPROVE AFFERENT & 1 Smoking cessation \\
VASOMOTION & 2 Calcium channel blockers \\
& 3 Consider sympathetic block \\
& (if response important, consider \\
& 4 sympathectomy) \\
& 5 Consider angiogram \\
& (tissue plasminogen activator if \\
& angiogram suggests amenable \\
& thrombosis) \\
& 1 Smoking cessation \\
& 2 Aspirin and/or other antiplatelet \\
IMPROVE MICROCIRCULATION therapy \\
& 3 Pentoxyifylline \\
& 4 Iloprost infusion \\
& 1 Smoking cessation \\
MINIMISE CONFOUNDING & 2 Use antibiotic early \\
FACTORS & 3 Correct hypoxia \\
& 4 Correct anaemia \\
& 5 Debridement/local wound care \\
& 6 Relieve pain and anxiety \\
IMPROVE VENOUS OUTFLOW & 1 Consider ketanserin \\
& 2 Elevate affected digit \\
& 3 Correct right heart failure \\
&
\end{tabular}


Treatment of vasculitis in the patient lacking other evidence is difficult to place in the variety of clinical approaches. We lack reliable laboratory markers for identification of unique subsets of patients in whom a specific aggressive therapy might be chosen. This would include the means for identification of appropriate candidates for thrombolytics such as tissue plasminogen activator; choice of subject for iloprost and other infused prostanoids; and perhaps a group of patients in whom treatment of vasculitis is appropriate.

Clinical Research Center, UMDNF-Robert Wood fohnson Medical School, New Brunswick, New fersey,

$U S A$

JAMES R SEIBOLD

1 Alexander E L, Firestein G S, Weiss J L, et al. Reversible cold-induced abnormalities in myocardial perfusion and function in systemic sclerosis. Ann Int Med 1986; 105: 661-8.

2 Furst D E, Davis J A, Clements P J, Chopra S K, Theofilopoulus A N, Chia $\mathrm{D}$. Abnormalities of pulmonary vascular dynamics and inflammation in early progressive systemic sclerosis. Arthritis Rheum 1981; 24: 1403-8.

3 Cannon P J, Hassar M, Case D B, Casarella W J, Sommers S C, LeRoy $\mathrm{E}$ C. The relationship of hypertension and renal failure in scleroderma (progressive systemic sclerosis) to structural and functional abnormalities of the renal cortical circulation. Medicine 1974; 53: 1 46.

4 Rodnan G P, Myerowitz R L, Justh G O. Morphologic changes in the digita arteries of patients with progressive systemic sclerosis (scleroderma) and Raynaud phenomenon. Medicine 1980; 59: 393-408.
5 Seibold J R. Serotonin and Raynaud's phenomenon. 7 Caradiovasc Pharmacol 1985; 7: S95-98.

6 Claman H N, Giorno R C, Seibold J R. Endothelial and fibroblastic activation in scleroderma. The myth of the "uninvolved skin." Arthritis Rheum 1991; 34: 1495-501.

7 Yamane $\mathrm{K}$, Kashiwagi $\mathrm{H}$, Suzuki $\mathrm{N}$, et al. Elevated plasma levels of endothelin-1 in systemic sclerosis. Arthritis Rheum 1991; 34: 243-4.

8 Marasini B, Cugno M, Agostini A. Plasma levels of tissue-type plasminogen activator and von Willebrand factor in patients with Raynaud's phenomenon. Arthritis Rheum 1991; 34: 255-6.

9 Wigley F M, Wise R A, Seibold J R, et al. Intravenous iloprost infusion in patients with Raynaud's phenomenon secondary to systemic sclerosis. A multi-center placebo-controlled double-blind study. Ann Intern Med 1994; 120: 199-206

10 Wigley F M, Seibold J R, Wise R A, McCloskey D A, Dole W P. Intravenous iloprost treatment of Raynaud's phenomenon and ischemic ulcers secondary to systemic sclerosis. $\mathcal{F}$ Rheumatol $1992 ; 19$ : 1407-13.

11 Kyle M V, Belcher G, Hazleman B L. Placebo controlled study showing therapeutic benefit of iloprost in the treatment of Raynaud's phenomenon. 7 Rheumatol 1992; 19: 1403-6.

12 Oddis C V, Eisenbeis C H, Reidbord H E, Steen V D, Medsger T A. Vasculitis in systemic sclerosis: association with Sjogren's syndrome and the CREST syndrome variant. F Rheumatol 1987; 14: 942-8.

13 Herrick A L, Oogarah P, Brammah T B, Freemont A J, Jayson M I V. Nervous system involvement in association with vasculitis and anticardiolipin antibodies in a patient with systemic sclerosis. Ann Rheum Dis 1994; 53: 349 .

14 Herrick A L, Oogarah P K, Freemont A J, Marcuson R, Haeny M, Jayson M I V. Vasculitis in patients with systemic sclerosis and severe digital ischaemia requiring amputation. Ann Rheum Dis 1994; 53: 323-6.

15 Helfrich D J, Banner B, Steen V D, Medsger T A. Normotensive renal failure in systemic sclerosis. Arthritis Rheum 1989; 32: 1128-34.

16 Boumpas D T, Chrousos G P, Wilder R L, Cupps T R, Balow J E. Glucocorticoid therapy for immune-mediated diseases: basic and clinical correlates. Ann Intern Med 1993; 119: 1198-208. 